\title{
Charge-spin excitations of the Ising-type fractional quantum Hall ferromagnets
}

\author{
Karel Výborný, ${ }^{1}$ Annelene F. Dethlefsen, ${ }^{2,3}$ Rolf J. Haug, ${ }^{2}$ and Arkadiusz Wójs ${ }^{4,5}$ \\ ${ }^{1}$ Fyzikální ústav Akademie věd České republiky, v.v.i., Cukrovarnická 10, CZ-16253 Praha 6, Czech Republic \\ ${ }^{2}$ Institut für Festkörperphysik, Leibniz Universität Hannover, Appelstraße 2, D-30167 Hannover, Germany \\ ${ }^{3}$ Solar Systems Pty. Ltd., 45 Grosvenor Street, Abbotsford, Victoria 3067, Australia \\ ${ }^{4}$ Instytut Fizyki, Politechnika Wrocławska, Wybrzeże Wyspiańskiego 27, 50-370 Wroclaw, Poland \\ ${ }^{5}$ TCM Group, Cavendish Laboratory, University of Cambridge, Cambridge CB3 OHE, United Kingdom
}

(Received 3 March 2009; revised manuscript received 29 May 2009; published 9 July 2009)

\begin{abstract}
We study the low-lying excitations from the fractional quantum Hall states at filling factors $2 / 3$ and $2 / 5$ and argue that the charge-carrying excitations involve spin flips, and, in particular, possibly more than one. Energies obtained by exact diagonalization and transport activation gaps measured over a wide range of magnetic fields are invoked. We discuss the relevance of the noninteracting composite-fermion picture where both fractions correspond to the same filling factor 2 of the composite fermions.
\end{abstract}

DOI: $10.1103 /$ PhysRevB.80.045407

PACS number(s): 73.43.-f, 75.10.Lp, 73.63.-b

In Jain's composite-fermion $(\mathrm{CF})$ picture, ${ }^{1}$ incompressible electron states $^{2}$ of the fractional quantum Hall (FQH) effect ${ }^{3}$ form pairs corresponding to the same magnitude of the effective magnetic field $B^{*}$ acting on the CFs. For example, the distinct FQH states at Landau-level (LL) filling factors $\nu$ $=2 / 5$ and $2 / 3$ both correspond to two effective LLs completely filled with the CFs, i.e., to the same effective filling factor $\nu^{*}=2$. These states are distinguished by the orientation of $B^{*}$ with respect to the real magnetic field acting on the electrons. Some properties of these FQH systems are known and can be understood using the mentioned analogy, for instance the Ising-like transition between ground states (GS) of different magnetization (paramagnetic and ferromagnetic), ${ }^{4,5}$ some other properties are known but the applicability of the composite-fermion picture is not established, like the existence of a half-polarized ground state ${ }^{6}$ (whose microscopic origin is still debated), ${ }^{7}$ and some are not explored yet. It was found in a previous study that the measured gaps for $\nu$ $=2 / 5$ and $2 / 3$ showed different dependences on magnetic field which was interpreted using different $g$ factors for CFs. ${ }^{8}$ However, no detailed calculations were undertaken at that time to understand this observed effect. Exactdiagonalization (ED) studies could clarify the underlying physics. Can charged excitations exist at these filling factors that carry large spin? If so, how far can these excitations be understood using the $\mathrm{CF}$ picture? Such excitations, identified as skyrmions, ${ }^{9}$ do exist at filling factor 1 and its CF counterpart $\nu=1 / 3$ which are Heisenberg-like quantum Hall ferromagnets $(\mathrm{QHF}){ }^{10}$ as shown by numerous experimental and theoretical arguments. ${ }^{11}$ They may be viewed as an extension of the idea of $\mathrm{FQH}$ quasiparticles involving single spin flip. ${ }^{12}$ Systems at filling factors $2 / 5$ and $2 / 3$ define a wider field for research, first because of their two possible GS (polarized and spin singlet), second because there are no analytical models for large spin quasiparticles such as skyrmions, and also because the similarity between $2 / 5$ and $2 / 3$ on the CF level can be tested. In this paper, we investigate the lowest excited states of these $\nu^{*}=2$ systems and present arguments for the spin flips being involved.

We begin by a recapitulation of known properties for $\nu$ $=2$ in Sec. I. The following section first deals with how this knowledge is transferred to the fractional fillings $2 / 5$ and $2 / 3$ and how this transfer is visualized using the noninteracting composite-fermion picture (NICF). An overview of numerical results from the ED for quasiexcitonic states is presented in Sec. II A and quasiparticles with more spin flips are reported in Sec. II B (with details and quantitative comparison to other theories ${ }^{13,14}$ given in Appendix). Finally in Sec. III, we analyze experimental results from activated transport gaps, compare them with the calculated energies, and propose which excitations were most likely to have been observed.

\section{EXCITATIONS IN A $\nu=2$ QHF}

In the absence of electron-electron interactions, there can be two different GS in the integer quantum Hall regime at $\nu=2$ : a fully polarized one and a spin-singlet one $\mathrm{e}^{15}$ as sketched in Fig. 1 including the spin $\sigma$ and Landau-level indices $n$ written as $(n, \sigma)$. The Zeeman spin splitting $E_{Z}$ $=g \mu_{B}|B|$ and the cyclotron energy $\hbar \omega$ determine which one will occur. Here, $B$ is the total magnetic field, $\mu_{B}$ is the Bohr magneton, $\omega=e\left|B_{z}\right| / m^{*}$ is the cyclotron frequency, $m^{*}$ is the effective mass, and $B_{z}$ is the magnetic-field component per-

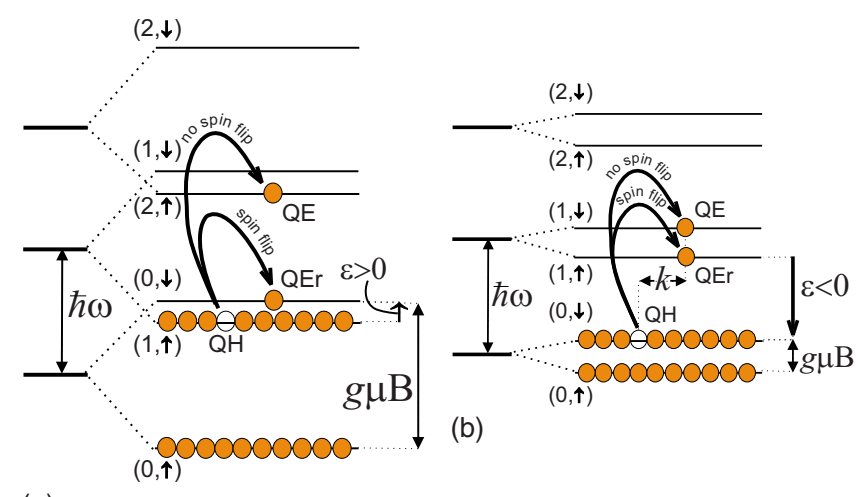

(a)

FIG. 1. (Color online) Two possible ground states at the integer filling factor $\nu=2$, (a) polarized and (b) spin-singlet one, and the lowest excitations in the noninteracting electron picture with and without spin flip. 
pendicular to the plane of the two-dimensional electron gas; filling factor can be defined as the number of electrons $N_{e}$ occupying area $A$ per magnetic-flux quantum piercing that area, $\nu=N_{e} /[A B /(h / e)]$. Since, in GaAs, $m^{*}$ is smaller than the vacuum electron mass by a factor of 0.067 and the Landé $g$ factor equals -0.44 we typically have $E_{Z}<\hbar \omega$ unless the magnetic field is strongly tilted $\left(B_{z} \ll B\right)$.

The transition occurs at $\epsilon=0$ if we define

$$
\epsilon=\frac{g \mu_{B}|B|-\hbar \omega}{E_{C}}, \quad E_{C}=\frac{e^{2}}{4 \pi \varepsilon \ell_{0}}
$$

which is also in absolute value the energy of the lowest spinflip excitation $\epsilon_{1}=|\epsilon|$, in Coulomb energy units $E_{C}$ (where $\varepsilon$ is the material permittivity and $\ell_{0}=\sqrt{e B / \hbar}$ is the magnetic length). Electron-electron interactions shift the transition to ${ }^{16}$ $\epsilon=-0.47$ since the exchange contribution reduces the total energy in the fully polarized state but the transition remains abrupt. ${ }^{15}$ As only the left-hand side of this GS transition condition depends on $B$, according to Eq. (1), the relative strength of interaction effects can be tuned, for example, if we change $B$ while keeping the filling factor constant.

In the spin-singlet ground state $(\uparrow \downarrow \mathrm{GS}, \epsilon<0)$, the absolutely lowest excitation always involves a spin flip and consists of a quasielectron with reversed spin (QEr) which leaves behind a quasihole $(\mathrm{QH})$. In the spin-polarized ground state $(\uparrow \uparrow \mathrm{GS}, \epsilon>0)$, the lowest excited state is either again QH/QEr with energy $\epsilon_{1}$ or, for large $\epsilon$, a quasielectron without spin reversal $(\mathrm{QE})$ becomes more favorable. Energy of such a state, a QE/QH pair, equals $\epsilon_{0}=\hbar \omega / E_{C}$ in Coulomb units, and does not depend on the (average) mutual distance $\Delta r$ of the $\mathrm{QH}$ and $\mathrm{QE}$ (or QEr for the other excitation) because we have disregarded the electron-electron interactions so far.

With this background, we wish to study the effect of interactions. As a consequence of the attractive Coulomb force between a $\mathrm{QE} / \mathrm{QEr}$ and a $\mathrm{QH}$, the excitation energy will generally increase with $\Delta r$ but exceptions due to the exchangeenergy contribution may occur at small distances. Total excitation energies $\epsilon^{\mathrm{exc}}$ in Coulomb units were expressed by Kallin and Halperin ${ }^{17}$ as a sum of the single-particle energy, $\epsilon_{0}$ or $\epsilon_{1}$ in our case, and interaction energy $\Delta \epsilon_{1}\left(k \ell_{0}\right)$, where $k=\Delta r / \ell_{0}^{2}$ is the momentum of the QE/QEr-QH pair. The dimensionless function $\Delta \epsilon_{1}$ depends on the ground state (polarized or singlet) and $(n, \sigma)$ of the level to which the QE has been excited (eventual spin flip involved). For example, the spin-flip (no-spin-flip) excitation energy from the $\uparrow \downarrow$ GS is shown in Fig. 4(a) of Ref. 17 where it is denoted by $\Delta E_{1}^{t}$ $\left(\Delta E_{1}^{s}\right)$.

\section{THEORETICAL EXPECTATIONS FOR FRACTIONAL FILLINGS}

The illustrative picture of noninteracting compositefermions maps both the (electronic) filling factor $\nu=2 / 5$ and $2 / 3$ to a single composite-fermion filling factor ${ }^{18}$ of $\nu^{*}=2$.

Let us briefly review this concept on the example of $\nu$ $=2 / 5$, where every two electrons share five magnetic-flux quanta. If each electron forms a quasiparticle composed of the electron and two flux quanta, called a $\mathrm{CF}$, there will be one $(5-2 \cdot 2)$ flux quantum for two CFs left giving rise to $\nu^{*}=2$. The remaining flux quantum is said to create effective field which, provided CF-CF interactions are not too strong, creates Landau levels, and the FQH effect at $\nu=2 / 5$ corresponds to the integer quantum Hall effect of CFs at $\nu^{*}=2$. Analogous simple calculation with $\nu=2 / 3$ gives minus one flux quantum $(3-2 \cdot 2)$ for two CFs, i.e., again $\nu^{*}=2$, and the same picture as for $\nu=2 / 5$, at least in the case of completely noninteracting CFs where the sense of the effective field is irrelevant.

This picture may be seen as a generalization of the fact that the Laughlin wave function, which is an excellent approximation $^{19}$ to the exact ground state of Coulombinteracting electrons at $\nu=1 / 3$, can be exactly reproduced by adding two flux quanta to each electron in a completely filled lowest Landau level, ${ }^{1}$ and corresponds thereby to $\nu^{*}=1$. Compared to results of exact diagonalization with electron systems ${ }^{20}$ and with experiments, ${ }^{5}$ the $\mathrm{CF}$ picture proved to be a very useful framework for understanding the FQH effect. However, the interactions between CFs need not always be weak, may lead to the formation of incompressible states at noninteger ${ }^{21} \nu^{*}$ or constitute the main part of excitation energies as shown in this paper. Here, we therefore use the picture of NICF only as a help in interpreting our exactdiagonalization results. In the Appendix, we turn our attention to quantitative aspects, and we compare our ED data with $\mathrm{CF}$ theories such as Jain's trial wave functions ${ }^{13}$ or bosonization formalism for spin waves. ${ }^{14}$

As detailed in Appendix A, both filling factors 2/3 and 2/5 exhibit two ground states, ${ }^{7,11}$ the $\uparrow \uparrow \mathrm{GS}$ and the $\uparrow \downarrow \mathrm{GS}$, in agreement with the NICF picture and the filling factor 2 scenarios in Figs. 1(a) and 1(b). An important difference to the integral quantum Hall effect is, however, that the $\mathrm{CF}$ Landau levels are "generated" by the electron-electron interaction. Following the standard procedure, ${ }^{22}$ all numerically calculated energies reported in this paper are obtained by diagonalizing the "ideal system" many-body Hamiltonian

$$
H=E_{C} \sum_{i<j} \frac{1}{\left|\vec{r}_{i}-\vec{r}_{j}\right| / \ell_{0}}
$$

with $N_{e}$ electrons $\left(i, j=1, \ldots, N_{e}\right)$ located at $\vec{r}_{i}$ within the lowest Landau level, i.e., neglecting the Landau-level mixing. In this paper, the energies will always be given in the Coulomb units $E_{C}$ as Eq. (1) suggests. Invoking the picture of NICF, both the CF cyclotron energy and exchange must therefore scale with $E_{C} \propto 1 / \ell_{0} \propto \sqrt{B}$ and cannot be separated one from another. In particular, it is not possible to control the CF-CF interaction independently from the CF cyclotron energy by a continuous parameter such as $\varepsilon$ (or $B$ ) in the integer regime. An analogy of the wave vector $k$ discussed above for two-particle systems (electron hole) can be defined also for many-body systems ${ }^{23}$ using relative translations of one electron with respect to the center of mass.

The CF cyclotron energy can be evaluated as $\left(E_{\mathrm{pol}}\right.$ $\left.-E_{\text {sing }}\right) /\left(N_{e} / 2\right)$, where $E_{\text {pol }}$ and $E_{\text {sing }}$ are the eigenenergies of Hamiltonian (2), i.e., at zero Zeeman energy, belonging to the $\uparrow \uparrow \mathrm{GS}$ and the $\uparrow \downarrow \mathrm{GS}$, respectively. ${ }^{5}$ The simple picture 
behind this procedure is that the difference in energies of the two states is due to $N_{e} / 2$ CFs located in the $(1, \uparrow)$ or $(0, \downarrow)$ CF Landau level. Such cyclotron energy need not be the same for different electron filling factors implying that the CF Landau-level ladder varies when its filling is changed.

In analogy to Eq. (1), we can define

$$
\epsilon^{\nu} \equiv\left[\mu_{B} g B-\frac{E_{\mathrm{pol}}-E_{\text {sing }}}{N_{e} / 2}\right] / E_{C} \equiv \eta-\Delta \epsilon^{\text {pol-sing }}
$$

for $\nu=2 / 3$ or $2 / 5$, together with Zeeman and Coulomb interaction energies $\eta$ and $\Delta \epsilon^{\text {pol-sing }}$ expressed in the Coulomb units $E_{C}$. If the ED is used to calculate the GS energies $E_{\mathrm{pol}}$, $E_{\text {sing }}$ in the $2 / 3$ or $2 / 5 \mathrm{FQH}$ system, these will naturally also contain any contribution that could be ascribed to $\mathrm{CF}-\mathrm{CF}$ interactions. In this way, $\epsilon^{\nu}=0$ will be the exact point of the phase transition between the polarized $\left(\epsilon^{\nu}>0\right)$ and the spinsinglet $\left(\epsilon^{\nu}<0\right)$ ground states.

The quantity $\Delta \epsilon^{\text {pol-sing }} \approx 0.01$ can be seen as a FQH analogy of the integer-filling regime $\hbar \omega / E_{C}+\epsilon_{t r} \approx 0.3 / 1.1$ of $\nu$ $=2$, where the value $\epsilon_{t r} \approx-0.47$ was discussed after Eq. (1) and $\hbar \omega / E_{C}$ used corresponds to magnetic fields 4/16 T. Transition to the polarized GS hence requires roughly 1-2 orders of magnitude larger Zeeman energy (in this range of magnetic fields) in the integer regime than in the $\mathrm{FQH}$ regime. Detailed ED data for $\Delta \epsilon^{\text {pol-sing }}$ of filling factors $2 / 3$ and $2 / 5$, given in Appendix A, hint at a similar value for both filling factors.

\section{A. Excitations}

Attempting to keep the analogy to the filling factor 2, the energy (in Coulomb units) of excitations from the FQH states at $\nu=2 / 3$ or $2 / 5$ could again formally be written as a sum of the "single CF" energy $\epsilon_{1}^{\nu}\left(\epsilon_{0}^{\nu}\right)$ and the CFmagnetoexciton energy $\Delta \epsilon_{1}^{\nu}\left(k \ell_{0}\right)$ for spin-flip (no-spin-flip) excitations. The dimensionless function $\Delta \epsilon_{1}^{\nu}\left(k \ell_{0}\right)$ would again depend on the type of excitation. However, the singleparticle excitation energies $\epsilon_{1}=\left|\eta-\Delta \epsilon^{\text {pol-sing }}\right|$ and $\epsilon_{0}$ $=\Delta \epsilon^{\mathrm{pol} \text {-sing }}$, evaluated in the $2 / 3$ or $2 / 5 \mathrm{FQH}$ system, entail also a part of the $\mathrm{CF}$ exchange energy which was exclusively contained in $\Delta \epsilon_{1}\left(k \ell_{0}\right)$ appropriate to $\nu=2$ excitations.

We therefore adopt a different framework for the $\mathrm{FQH}$ excitations. Dispersion branches sketched in Fig. 2 are based directly on the eigenenergies of the full Hamiltonian (2) for $\nu=2 / 3$ and $2 / 5$ taken with respect to the GS energy at $\eta=0$. The no-spin-flip (single-spin-flip) modes are shown by solid (dashed) lines. In Fig. 2(a), they are the energy intervals from the $\uparrow \uparrow \mathrm{GS}$ to the lowest excited state with total spin $S$ $=N_{e} / 2\left(S=N_{e} / 2-1\right)$. Sketches in Fig. 2(b), on the other hand, visualize the lowest excitations from the $\uparrow \downarrow$ GS within the $S=0(S=1)$ subspace. They could formally be interpreted in terms of expressions $\Delta \epsilon^{\text {pol-sing }}+\Delta \epsilon_{1 \text {,singlet,no s.f. }}^{\nu}\left(k \ell_{0}\right)$ [and $\left.\Delta \epsilon^{\text {pol-sing }}+\Delta \epsilon_{1 \text {,singlet,s.f. }}^{\nu}\left(k \ell_{0}\right)\right]$ that give the zero (and single) spin-flip excitation energies from the spin-singlet GS at $\nu$ $=2 / 3$ or $2 / 5$. Regarding the polarized GS and Fig. 2(a), the corresponding expressions are $\Delta \epsilon^{\text {pol-sing }}+\Delta \epsilon_{1 \text {,no s.f. }}^{\nu}\left(k \ell_{0}\right)$ [and $\left.-\Delta \epsilon^{\text {pol-sing }}+\Delta \epsilon_{1 \text {,s.f. }}^{\nu}\left(k \ell_{0}\right)\right]$, assuming that the CF LLs are equidistant. Here, we must bear in mind that (i) so as to get the total excitation energies, positive Zeeman energy $\eta$ has to be added for the spin-flip modes shown in Fig. 2 and (ii) sizable
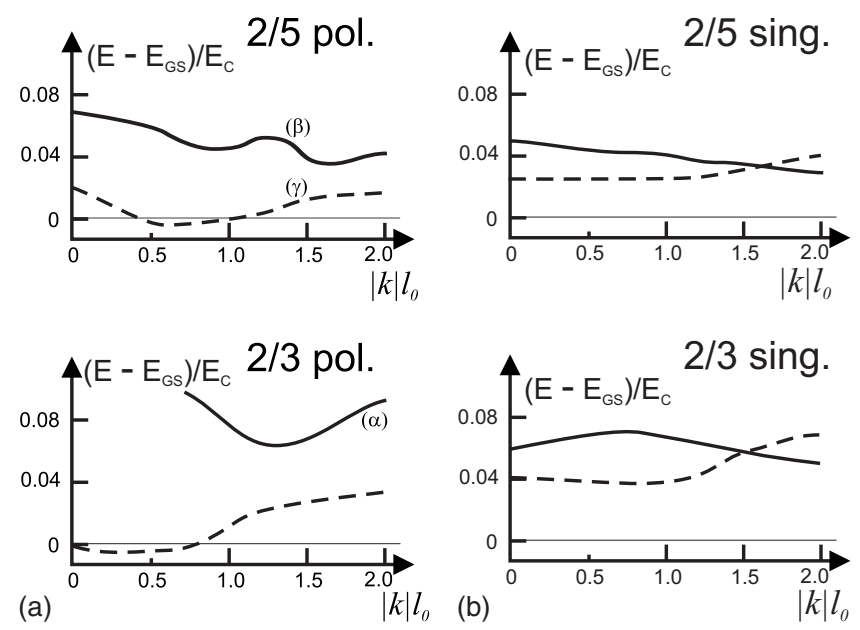

FIG. 2. Schematics of dispersion branches of the lowest excitations from the (a) polarized, (b) spin-singlet ground states corresponding to the CF filling factor $\nu^{*}=2$ (i.e., $\nu=2 / 5$ and $\nu=2 / 3$ ). Solid (dashed) lines show no-spin-flip (single-spin-flip) excitations. The line $(\alpha)$ is based on our earlier data (Ref. 24) and all other sketches on the ED data detailed in Appendix B. Employing diverse approaches, some of the dispersions have already been estimated before: $(\alpha)($ Ref. 25), $(\beta)$ (Ref. 26), and ( $\gamma)($ Ref. 13).

value of $\eta$ (relative to $\Delta \epsilon^{\text {pol-sing }}$ ) is needed for the polarized ground state to become favored over the spin-singlet one.

Direct numerical data underlying Fig. 2 are presented in Appendix B. We now semiquantitatively discuss the particular excitations in the perspective of CF-magnetoexcitons analogous to $\mathrm{QE}-\mathrm{QH}$ or $\mathrm{QEr-QH}$ pairs in the integer quantum Hall regime, illustrated in Fig. 1.

\section{Excitations from the polarized states}

Despite the close relationship of $\nu=2 / 5$ and $2 / 3$ in the NICF picture, the dispersion branches, solid lines in Fig. 2(a), are qualitatively different. This result is not surprising. The excitation in the $\nu=2 / 5$ state, interpreted using Fig. 1(a), consists of a particle promoted to the $(2, \uparrow) \mathrm{CF}$ Landau level interacting with a hole in the $(1, \uparrow)$ level and this interpretation agrees with the CF trial-wave-function calculation. ${ }^{27}$ Starting with intuitive electrostatics, the interaction energy as a function of the mutual distance between a quasiparticle and a quasihole will be minimised if their overlap is maximal. Wave functions of a particle or hole in the second and first Landau level have two extrema (one extremum) and it is likely that combining two such objects may lead to more than just one local minimum in $E\left(k \ell_{0}\right)$. Now, let us turn our attention to $\nu=1$ as an analogy of the $\nu=1 / 3$ system and also the $\nu=2 / 3$ polarized system by virtue of the particle-hole symmetry. $^{22}$ The (identical) excitation spectra of the $\nu=1 / 3$ and $2 / 3$ fully polarized systems contain the magnetoroton mode which gives the lowest excitation at nonzero $k$ as expected for a $(1, \uparrow)$ particle interacting with a $(0, \downarrow)$ hole. The real charge densities of quasiparticles and quasiholes at $\nu$ $=1 / 3$ and $2 / 5$ (see Appendix D) qualitatively support the validity of this interpretation.

Spin-flip excitations from the polarized states are shown by dashed lines in Fig. 2(a). The bare fact that their energy 
TABLE I. Estimates of large-wave-vector limits of the excitation energies in the Coulomb units $E_{C}$ corresponding to modes sketched in Fig. 2. The underlying ED data can be found in the Appendix.

\begin{tabular}{ccccc}
\hline \hline \multicolumn{2}{c}{ Polarized ground state } & \multicolumn{2}{c}{ Spin singlet ground state } \\
\hline & $2 / 5$ & $2 / 3$ & $2 / 5$ & $2 / 3$ \\
no spin flip & 0.04 & 0.10 & 0.03 & 0.05 \\
single spin flip & 0.01 & 0.03 & 0.04 & 0.06 \\
\hline \hline
\end{tabular}

can be negative is not surprising because of the negative single CF contribution corresponding to the promotion from $(1, \uparrow)$ to $(0, \downarrow)$ CF Landau level under zero Zeeman energy. Regarding the values of $\Delta \epsilon^{\text {pol-sing }}$, the negative-energy portions of $E\left(k \ell_{0}\right)$ are in fact rather shallow. Within the CF framework, this would again point at sizable CF exchangeenergy contribution.

A shallow minimum of the $\nu=2 / 5$ excitation spectrum at roughly $k \ell_{0} \sim 0.5$, Fig. 2(a), agrees with the model CF trialwave-function calculation ${ }^{13,28}$ and, again, with the picture of a $(1, \uparrow)$ particle interacting with a $(0, \downarrow)$ hole explained above for no-spin-flip excitations. On the other hand, the $\nu=2 / 3$ spin-flip mode dispersion shows only very little signs of such minimum and rather resembles the spin wave of the $\nu=1 / 3$ system. $^{24}$ This is somehow surprising as the particle-hole symmetry between $\nu=1 / 3$ and $2 / 3$ cannot be expected to hold as soon as the states are not fully spin polarized.

Note that energies of the spin-flip and no-spin-flip excitations at $k \rightarrow 0$ in Fig. 2(a) differ by significantly more than $2 \Delta \epsilon^{\text {pol-sing }} \leqq 0.02$; Fig. 1(a) makes it clear why such an expectation may be in place if $\mathrm{CF}$ interactions are neglected. This is one example of a marked quantitative inconsistency of the NICF picture. Invoking the analogy to $\nu=2$ this means that the $\mathrm{CF}$ exchange energies are at least comparable to the single-particle energies.

\section{Polarized states-summary}

Despite some inconsistencies with the NICF, the agreement between the ED and energies obtained from the CF trial wave functions ${ }^{13,27}$ suggests that the lowest excitations are well-defined modes whose wave vector $k$ is proportional to the spatial quasiparticle-quasihole separation. Rough estimates of the $k \rightarrow \infty$ energies are summarized in Table I. There are spin-flip excitations from the polarized GS's that have negative energy if we do not count the Zeeman contribution and some of them can be seen in Fig. 2(a). Consistently, the polarized GS has a higher energy than the spin-singlet GS when Zeeman energy is zero (see Appendix A).

\section{Excitations from the spin-singlet states}

A very apparent feature of both $\nu=2 / 3$ and $2 / 5$ dispersions in Fig. 2(b) is that the no-spin-flip and single-spin-flip modes are energetically much closer to each other than for the polarized states in Fig. 2(a). This agrees with the NICF picture where the single-particle energy of both types of excitations is equal up to Zeeman energy. The dispersions of the spin-flip modes seem similar to those of the polarized ground state counterparts up to an overall shift, compare the dashed lines in Figs. 2(a) and 2(b). This again agrees with the NICF picture, in the analogy of Fig. 1. Both types of excitations represent a $\mathrm{QH} / \mathrm{QEr}$ pair, only the role of the $(0, \downarrow)$ and $(1, \uparrow)$ CF Landau levels exchanges between excitations from the polarized and from the spin-singlet ground states. The overall energy shift should then be twice larger than the $\mathrm{CF}$ cyclotron energy. The inferred value of this single-particle energy, 0.025 for $\nu=2 / 3$ and 0.02 for $\nu$ $=2 / 5$ is about twice as large than expected from the GS energies $\left(\Delta \epsilon^{\text {pol-sing }}\right.$, (see Appendix A) and can thus be accommodated into the NICF picture, however, with sizable $\mathrm{CF}$ exchange-energy corrections.

Contrasting with our results, the approach based on the single-mode approximation, ${ }^{29}$ yields qualitatively different results for the $\nu=2 / 5$ singlet state. The no-spin-flip excitations have unrealistically high energy and so do the singlespin-flip excitations for larger values of $k$. The latter, associated with a spin mode (rather than with a Goldstone mode), however have the energy quite close to the ED result for $k=0$.

Based on the available ED data it is not possible to conclude if the energy dispersions eventually bend upward for large $k$. In illustrative terms, the energy cost of separating oppositely charged particle and hole may or may not be compensated by the gain in the exchange energy. Estimates of the limiting values of excitation energy based on this limited set of data are summarized in Table I.

\section{B. More spin flips}

We will now focus only on excitations from the spinpolarized GS's. However, in Appendix C we also present some results for the spin-singlet GS's. Although we are now dealing with Ising QHF, ${ }^{11}$ we will begin by recalling the situation in different systems.

Heisenberg-type quantum Hall ferromagnets can be expected to ${ }^{9}$ and were shown to ${ }^{30}$ support skyrmionic excitations. However, in small finite systems accessible by the ED, a "magnetoexciton" consisting of a skyrmion/antiskyrmion pair rather than $\mathrm{QE} / \mathrm{QH}$ cannot be resolved. We therefore adopt an alternative approach which we used before to study $\nu=1 / 3$ systems $:^{24}$ we started with $\epsilon^{\infty}$, the large $k$ limit of the QE/QH magnetoexciton mode at $\nu=1 / 3$, where the interaction between the particle and the hole vanishes. Then we calculated the spectrum of a $\nu=1 / 3$ system with one extra QE and studied how the particle can lower its energy by creating a collective state with other "background" particles associated with the decrease in the total spin of the system. Depending on the ratio of Zeeman and Coulomb energies, the optimum state was chosen and its energy was added to 
TABLE II. Coulomb energy gains in a $\nu=2 / 5$ or $2 / 3$ system with one extra quasiparticle (quasihole) when spin flips are allowed. Energies are given in the Coulomb units $E_{C}$, full numerical data are shown in Appendix C.

\begin{tabular}{ccccccc}
\hline \hline \multicolumn{2}{c}{ Quasiparticle excitations } & & \multicolumn{2}{c}{ Quasihole Excitations } \\
\cline { 1 - 2 } \cline { 5 - 6 } Spin flips & $2 / 5$ & $2 / 3$ & & $2 / 5$ & $2 / 3$ \\
\hline 1 & -0.049 & -0.053 & & -0.017 & -0.023 \\
2 & -0.010 & -0.015 & & -0.010 & -0.016 \\
\hline \hline
\end{tabular}

$\epsilon^{\infty}$. The same procedure was repeated with the $\mathrm{QH}$ and we finally arrived at the corresponding excitation involving several spin flips. difference between no-spin-flip and single-spin-flip values of $\epsilon^{\infty}$ and compare it to the energy difference of a $\mathrm{QE}$ and a QEr. The latter value obtained from the spectrum of a $\nu$ $=1 / 3$ system with one extra $\mathrm{QE}(0.0385)$ passes this check reasonably (compare Table II in Ref. 24).

We now turn to the $\nu=2 / 5$ and $2 / 3$ systems, keeping in mind that these will suffer more from the finite size of the system studied by the ED than the $\nu=1 / 3$ one. We only discuss excitations from the spin-polarized ground states. The consistency check described in the previous paragraph is roughly fulfilled, but it is remarkable that the difference of extrapolated guesses for the no-spin-flip and single-spin-flip excitations in Table I underestimates the QE/QEr spin-flip energy (of Table II) found at $\nu=2 / 5+\mathrm{QE}(-0.049)$ while it overestimates this energy at $\nu=2 / 3+\mathrm{QE}(-0.053)$. The likely reason is that the $\nu=2 / 5$ no-spin-flip energy and the $\nu=2 / 3$ single-spin-flip energy are in fact somewhat larger than the estimates in Table I (compare the full numerical data in Appendix B showing how values in Table I were obtained). Note that in Table II and Appendix C, we only employ the spherical geometry ${ }^{24}$ as the torus geometry is not suitable for studies of states with a single small skyrmion. ${ }^{31}$

More spin flips beyond $\mathrm{QE} \rightarrow \mathrm{QEr}$ can further decrease the energy of the system as shown in the quasiparticle excitation section of Table II. In a $\nu=1$ system, which is a Heisenberg QHF, similar states occur that can be associated with the smooth spin textures called skyrmions, known to be solutions of certain Lagrangians [some related to the QHE (Ref. 9) and some not]. There is a clear similarity between the $\nu=1+\mathrm{QE}$ and $\nu=1 / 3+\mathrm{QE}$ spectra ${ }^{32}$ suggesting that also the Heisenberg QHF at $\nu=1 / 3$ supports skyrmions. However, since $\nu=2 / 5$ and $2 / 3$ are Ising rather than Heisenberg ferromagnets, the question on the nature of the two- and more-spin-flips excitations here remains open. We visualize these states as, e.g., trions (two QEr's and one QH) as shown in Fig. 3(a), and present arguments in Appendix C why this
A check of consistency of this procedure is to take the

visualization is reasonable. Nevertheless, however analogous these $C F$ sketches are to the situation at $\nu=1 / 3$ plus QEr, we cannot draw the conclusion that the electronic wave functions will be similar (and have, for example, skyrmionlike spin texture). Specifically, it is not clear whether the spin texture size of $\nu=2 / 5+\mathrm{QE}$ would grow with decreasing Zeeman energy as it is the case at $\nu=1 / 3+\mathrm{QE}$. Systems accessible to the ED are too small to answer this question fully, yet as we argue below, more than a single spin flip may realistically occur.

Similar visualization applies also to $\nu=2 / 5$ and $2 / 3$ plus a quasihole, see Fig. 3(b), energies are summarized in the right part of Table II and the original data underlying Table II are shown in Appendix C.

Lowest excitation energies as shown in Fig. 4, can readily be evaluated by combining Tables I and II. The ratio of Zeeman energy to the Coulomb energy determines which of the spin-flip excitations has the lowest energy. Recalling the definition in Eq. (3),

$$
\eta=\frac{\mu_{B} g B}{E_{C}} \approx 0.006 \sqrt{B[\mathrm{~T}]},
$$

the first spin flip in the lowest excitation (imagine starting in $B \rightarrow \infty$, i.e., $\eta \rightarrow \infty)$ of, for instance the $\nu=2 / 5$ system, will occur for $\eta \leq 0.049$, see Table II. Below the corresponding field $(\sim 65 \mathrm{~T})$, the $\mathrm{QH} / \mathrm{QEr}$ excited state will be preferred to the $\mathrm{QH} / \mathrm{QE}$ one because the gain in the Coulomb energy overweights the loss of the Zeeman energy. The second transition occurs at $\eta \leq 0.017(B \sim 8 \mathrm{~T})$ where it becomes favorable to change the spin by one also in the hole part of the excited state as seen in the top left panel of Fig. 4. This state would be a $\nu=2 / 5$ analogy of the smallest antiskyrmion reported in the $\nu=1 / 3$ system. ${ }^{24}$ However, we stress that $\nu$ $=2 / 5$ is an Ising rather than Heisenberg QHF, so that there is no reason to link its excitations to skyrmions. ${ }^{9}$ Transitions within the lowest excitation to states containing more spin flips are precluded by the excitation energy dropping below zero at $B \sim 5 \mathrm{~T}$, a hallmark of the approaching transition between $\uparrow \uparrow$ GS and $\uparrow \downarrow$ GS transition (which should, according to data in Appendix A occur for $B \sim 1 \mathrm{~T}$ ). For $\nu=2 / 3$ (top right panel of Fig. 4), we obtain a slightly different picture. The excitation energies and transition fields to excitations containing more spin flips are generally higher (see Table I). We observe a transition to as many as three-spin-flip excitation already at $B \sim 7 \mathrm{~T}$. Based on available ED data (see Appendix $\mathrm{C}$ ), we are unable to say whether additional spin flips will be preferred at lower magnetic fields and/or a region of instability toward spin-flip excitations occurs before the $\uparrow \uparrow \mathrm{GS} / \uparrow \downarrow \mathrm{GS}$ transition.

We conclude this section by presenting a tentative phase diagram of the lowest excitations in $\nu=2 / 5$ and $2 / 3$ systems
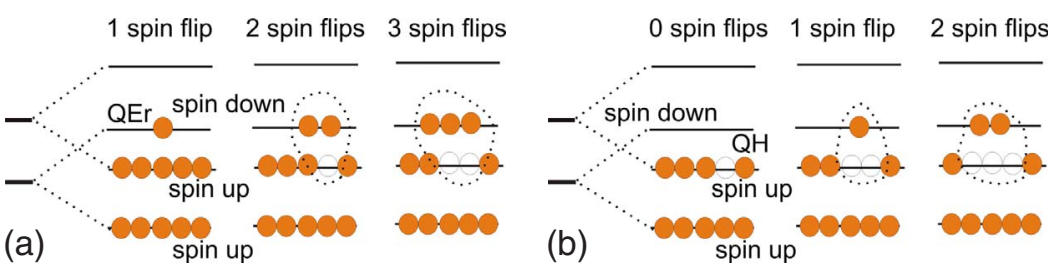

FIG. 3. (Color online) A special class of lowenergy states in a fully polarized $\nu=2$ system plus (a) one $\mathrm{QEr}$ and (b) one QH. We use these sketches in the CF sense to visualize the lowestenergy states with various total spin in $\nu=2 / 5$ or 2/3 plus one $\mathrm{QEr}$ or $\mathrm{QH}$. 

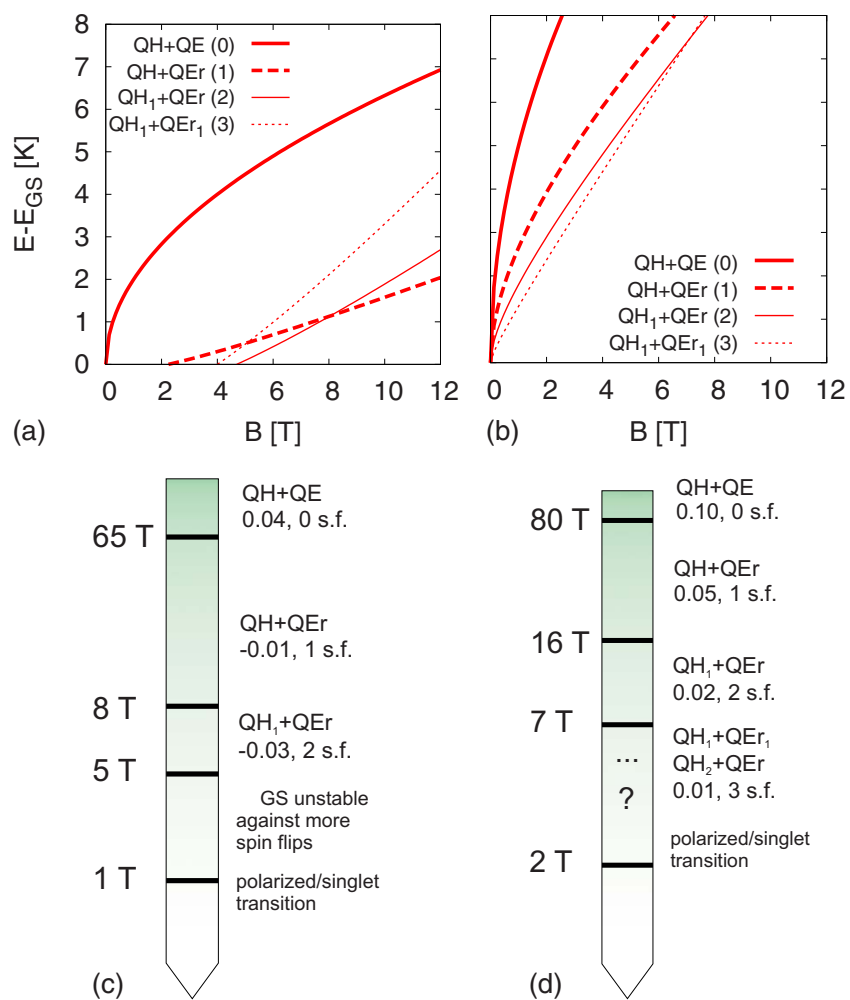

(b)

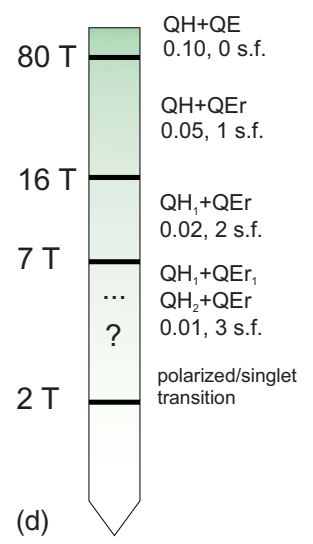

FIG. 4. (Color online) Above: Excitation energies in an ideal disorder-free system based on the QE/QH mode energy of Table I and spin-flip energies of Table II. Total number of spin flips involved is given in parentheses. Below: Fields corresponding to the spin transitions in the lowest excited state. Corresponding Coulomb energies of the excitations and total number of spin flips are given on the right. QH/QE/QEr denotes a quasihole/quasielectron/ quasielectron with reversed spin, and if any of the objects involves more spin flips, it is indicated by the subscript. Larger scale numerical calculations are needed to find out whether excitations with more spin flips may occur (in range denoted by “?”).

in the lower part of Fig. 4. We stress that the data is not extrapolated to very large systems and refers only to ideal systems (zero width, no LL mixing). It is likely that, after such treatment, the transition fields would generally decrease (and maybe even change order) just as in the $\nu=1 / 3$ case $^{24}$ which is easier to study. However, given the large magnitude of the first few transition fields, it is likely that the lowest excitations will still involve one or two spin flips close to the polarized-singlet ground-state transition.

\section{ANALYSIS OF EXPERIMENTS}

To complement our theoretical analysis, we also performed activation transport measurements (described recently in a greater detail in Ref. 33) on a particularly designed sample with the electron concentration varied in a wide range from 2 to $12.9 \times 10^{10} \mathrm{~cm}^{-2}$. This allowed us to determine the excitation gaps at fixed $\nu=2 / 3$ or $2 / 5$ as a function of magnetic field in the range of $B=2-12 \mathrm{~T}$.

One possible approach to analyze the experimentally determined gaps at various magnetic fields ${ }^{24}$ is based on the observation that Zeeman energy term $H_{Z}$ commutes with

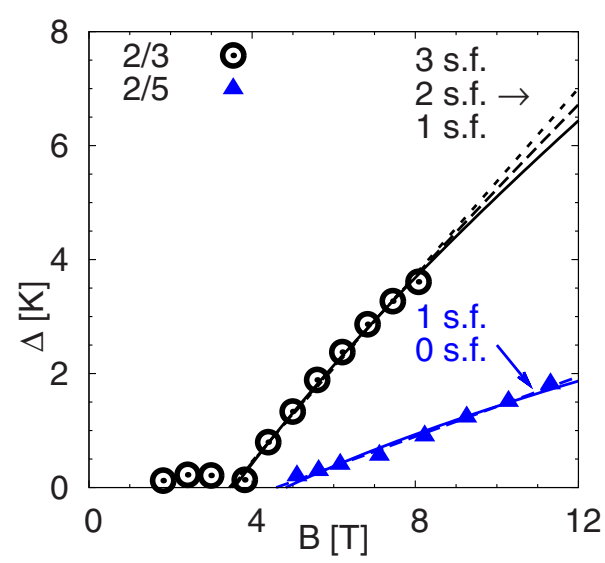

FIG. 5. (Color online) Experimental transport activation gaps from Ref. 33 with fits assuming $0,1,2$, or 3 spin flips (for $\nu=2 / 3$ ) and 0 or 1 spin flip (for $\nu=2 / 5$ ). Corresponding fit parameters of Eq. (5) are summarized in Table III.

Hamiltonian (2), so that the energy difference $E^{\text {exc }}$ (in Kelvin) between any two eigenstates of $\mathrm{H}+\mathrm{H}_{Z}$ has the form

$$
E^{\operatorname{exc}}(B)=\epsilon_{C} \cdot 50 \sqrt{B[\mathrm{~T}]}+\Delta s_{z} \cdot 0.3 \cdot B[\mathrm{~T}]-E_{d} .
$$

Here, $\epsilon_{C}$ is the Coulomb energy in Coulomb units $E_{C}$ $=e^{2} /\left(4 \pi \varepsilon \ell_{0}\right)$ (stemming from $\left.H\right), \Delta s_{z}$ is the integer number of spin flips involved in the excitation, and $-E_{d}$ has been added as a phenomenological disorder-induced gap reduction ${ }^{24,34}$ in Kelvin. We fit Eq. (5) to experimental data for several reasonable values of $\Delta s_{z}$, choose the best fit according to criteria discussed below (value of $E_{d}$, theoretical expectations on $\Delta s_{z}$, quality of fit) and compare the obtained $\epsilon_{C}$ with its theoretical value.

\section{Filling factor $2 / 5$}

The measured gaps are shown by filled triangles in Fig. 5 and results of the fitting are summarized in Table III. We obtain negative values of $-E_{d}$ for $\Delta s_{z}=2$ and 3 and because we expect the disorder to reduce rather than to enlarge the gaps, we are left with only two options. Although the quality of the fit (represented by the smaller sum of squared deviations) is better for $\Delta s_{z}=1$, the difference to the $\Delta s_{z}=0$ fit is visually almost imperceptible as demonstrated in Fig. 5. As a decisive argument we therefore take the theoretically calculated Coulomb energy gain for the first spin flip which is about twice the energy cost in Zeeman energy in ideal systems at the magnetic fields studied, see Table II and Eq. (4). Reductions in the gap at $\nu=1 / 3$ due to Landau-level mixing and finite thickness analyzed in the same sample are small ${ }^{24}$ and it is therefore likely that the observed excitation at $\nu$ $=2 / 5$ involves a $\mathrm{QH}$ and a $\mathrm{QEr}$. The value of $\epsilon_{C}=-0.001$ obtained by fitting for $\Delta s_{z}=1$ (see Table III) lies in between the theoretical estimates of $\mathrm{QEr} / \mathrm{QH}$ magnetoexciton $(0.01$, Table I) and of $\mathrm{QE} / \mathrm{QH}$ magnetoexciton plus a $\mathrm{QE} \rightarrow \mathrm{QEr}$ spin flip (-0.01, Tables I and II). Given that we omitted all the usual refining procedures with the ED data ${ }^{24}$ (careful thermodynamical extrapolation, finite thickness, etc.), such an agreement is satisfactory. A comment is due to the obtained gap reduction because the values $-E_{d}$ in Table III vary 
TABLE III. Fitting parameters for Fig. 5.

\begin{tabular}{lccccc}
\hline \hline & $\nu=2 / 5$ & & & $\nu=2 / 3$ & \\
\cline { 5 - 6 }$\epsilon_{C}$ & $-E_{d}$ & $\begin{array}{c}\# \text { of } \\
\text { spin flips }\end{array}$ & $\epsilon_{C}$ & $-E_{d}$ & $\begin{array}{c}\text { \# of } \\
\text { spin flips }\end{array}$ \\
\hline 0.029 & 3.2 & 0 & 0.077 & 7.5 & 0 \\
-0.001 & 1.2 & 1 & 0.052 & 5.9 & 1 \\
-0.030 & -0.9 & 2 & 0.026 & 4.4 & 2 \\
-0.060 & -3.0 & 3 & 0.001 & 2.9 & 3 \\
\hline \hline
\end{tabular}

rather strongly over the number of spin flips. We do not claim here that if we had surveyed a larger range of magnetic fields and had been able to observe the spin transitions predicted in Fig. 4, the parameter $-E_{d}$ would vary as much as given in Table III (some variation is possible, though, as we argued in Fig. 9 of Ref. 24). These values are only meant for the purpose of selection of the one particular type of excitation observed in our experiment.

\section{Filling factor $2 / 3$}

The empty circles in Fig. 5 show that the excitation gap closes at $B \approx 4 \mathrm{~T}$ and opens again at lower magnetic fields. We interpret this as the theoretically ${ }^{35}$ and experimentally ${ }^{4}$ known transition between the polarized and unpolarized incompressible ground states. We now focus on the part of data above this threshold. Fitting this data by expression (5) with $\Delta s_{z}=0,1,2,3$ produces visually nearly equally good results, see Fig. 5, the obtained values of $\epsilon_{C}$ and $E_{d}$ are summarized in Table III. The quality of the fits gradually decreases from $\Delta s_{z}=0$ (where $\epsilon_{C}$ is the largest) to $\Delta s_{z}=3$ (where $\epsilon_{C}$ is almost zero) corresponding to the fact that the measured $B$ dependence is slightly sublinear.

Compared to $\nu=2 / 5$, the values of $-E_{d}$ found by fitting are rather large. The gap reductions need not be the same for different filling factors (the quasiparticles involved in the excitations are not the same) but it seems unlikely that there would be a factor of 5 difference as between the $\Delta s_{z}=1$ fit of $\nu=2 / 5$ and $\Delta s_{z}=0$ of $\nu=2 / 3$. The $\Delta s_{z}=2$ excitation seems to be a good compromise between this and the other aspects: the quality of the fit and the calculated Coulomb energy gains in Table II suggesting more spin flips than for $\nu=2 / 5$ (see also Fig. 4). The fitted value $\epsilon_{C}=0.026$ for $\Delta s_{z}=2$ compares well with the theoretical estimates based on Tables I and II (0.01 from QEr/QH energy plus single spin flip QH $\rightarrow \mathrm{QH}_{1}$ or 0.02 from $\mathrm{QE} / \mathrm{QH}$ energy plus two spin flips $\mathrm{QE}$ $\rightarrow \mathrm{QEr}$ and $\mathrm{QH} \rightarrow \mathrm{QH}_{1}$ ). The analysis therefore suggests that the $\nu=2 / 3$ excitation observed in experimental data of Fig. 5 consists of a QEr and a quasihole with one additional spin flip, $\mathrm{QH}_{1}$.

\section{Comment on the $2 / 3$ singlet state}

It is not reasonable to use Eq. (5) for fitting the small gaps $\sim 0.5 \mathrm{~K}$ observed in experiment shown in Fig. 5 (again empty circles, now below $4 \mathrm{~T}$ ). They will most likely be strongly influenced by disorder and also by Landau-level mixing because the magnetic field is relatively low. Theoreti- cally expected Coulomb energy of excitations (see Table I) is almost an order of magnitude larger $(\sim 4 \mathrm{~K}$ at $2.5 \mathrm{~T})$. Spin flips are preferred by the Zeeman energy and will reduce this value, however at the cost of Coulomb energy comparable to the Zeeman energy gain; according to Fig. 13, for instance the cost of an additional spin flip of a $\mathrm{QH}, \sim 1 \mathrm{~K}$, is even slightly larger than the potential gain. (Here, we note that in the NICF, the spin-flip and no-spin-flip excitations should differ only by the Zeeman energy and thus spin flip should always for $\eta \neq 0$ be preferred.) It is likely that the theoretical estimate would significantly decrease after the mentioned refining procedures ${ }^{24}$ among which especially the $1 / N_{e} \rightarrow 0$ extrapolation would require huge computational effort. However, we cannot exclude that other states than effective single-quasiparticle excitations become important close to the GS transition. ${ }^{11}$

\section{CONCLUSION}

Different possibilities of lowest energy excitations at filling factor $\nu=2$ were reviewed in the noninteracting particle picture. Subsequently, the parallel to the fractional filling factors of $2 / 3$ and $2 / 5$ was drawn, motivated by the noninteracting composite-fermion picture. The exact-diagonalization results show that the analogy is qualitatively correct for the polarized ground state: the lowest excitation may involve zero or one spin flip depending on the ratio of Zeeman and Coulomb energies. However, in order to quantitatively compare the energies of various excitations, the noninteracting composite theory is not sufficient. If we insist on this framework we must ad hoc assume different exchange energies of composite fermions in different Landau levels.

The exact diagonalization shows that excitations with more spin flips are possible at $\nu=2 / 3$ and 2/5 although an interpretation of such states is not available (such as skyrmions at filling factors 1 or $1 / 3$ ). It would be interesting to see if ideas on spin textures developed for Heisenberg ferromagnets $(\nu=1 / 3)$ can also be extended to Ising ferromagnets $(\nu=2 / 5)$. For ideal systems, a theoretical summary of which excitations are expected to be the lowest ones at which magnetic fields was given in Fig. 4. Excitations with one or more spin flips are likely at experimentally relevant fields around $10 \mathrm{~T}$. Based on our analysis of measured transport activation gaps we suggest that a QEr/QH pair was excited at $\nu=2 / 5$ and a $\mathrm{QH}$ with an additional spin flip was involved for $\nu$ $=2 / 3$. 
TABLE IV. Coulomb energy gain of the spin-singlet GS with respect to the spin-polarized GS calculated as $\Delta \epsilon^{\mathrm{pol}-\text { sing }}=\left(E_{\mathrm{pol}}-E_{\mathrm{sing}}\right) \cdot E_{C}^{-1} /\left(N_{e} / 2\right)$, see Eq. (3).

\begin{tabular}{ccccccc}
\hline \hline & $2 / 5$ & & & \multicolumn{2}{c}{$2 / 3$} \\
\cline { 1 - 2 } \cline { 5 - 6 }$\#$ el. & Torus & Sphere & & $\#$ el. & Torus & Sphere \\
\hline 6 & 0.0064 & & 8 & 0.0079 & 0.0038 \\
8 & 0.0066 & 0.0107 & & 10 & 0.0084 & 0.0047 \\
& & & $\infty$ & 0.0073 & 0.0097 \\
\hline
\end{tabular}

Given the limits of computational possibilities and capabilities to precisely model the particular disorder occurring in a specific experimental sample, it seems unlikely that the precise number of spin flips involved in excitations at filling factors $2 / 3$ or $2 / 5$ can be determined with certainty unless a clear transition in the excited states is observed in some sample using a broad range of magnetic fields such $\mathrm{as}^{24}$ at $\nu=1 / 3$.

\section{ACKNOWLEDGMENTS}

Numerous advice of Mark O. Goerbig, Kentaro Nomura, and Daniela Pfannkuche proved helpful as well as support by Czech institutions under Grant Nos. AV0Z10100521, LC510, KAN400100652, and KJB100100802, and the Polish MNiSW under Grant No. N202-071-32/1513. A.W. acknowledges funding from an EU Marie Curie Program.

\section{APPENDIX A: NUMERICAL DATA-SINGLET- AND POLARIZED-GROUND-STATE ENERGIES}

Not counting the Zeeman energy, the lowest state in the $S=0$ sector $(\uparrow \downarrow$ GS $)$ is lower in energy than the fully polarized ground state $(\uparrow \uparrow$ GS) both for $\nu=2 / 3$ and $2 / 5$. This fact was noticed already in $1983:^{36}$ the fully polarized GS at $\nu$ $=2 / 5$ has a higher energy than the spin-singlet (332) Halperin wave function ${ }^{35}$ which has a very high overlap with the ( $\uparrow \downarrow$ GS) calculated by the ED. By turning the Zeeman energy on, the polarized state can be made the absolute ground state as expressed by the condition $\epsilon^{\nu}>0$ of Eq. (3). Here we summarize the differences in numerically calculated Coulomb energies of the polarized/singlet-GS pairs. Within the NICF picture, the energy difference in Coulomb units per $N_{e} / 2$ particles, $\Delta \epsilon^{\text {pol-sing }}$, corresponds to the energy separation between the lowest and the first CF LL as visualized in Fig. 1.

The energy differences in Coulomb units $E_{C}$ are of the order of $\approx 0.01$ for both fractions. Table IV summarizes available ED data. The $\nu=2 / 3$ values extrapolated to large systems were taken from Ref. 11. In spite of the missing extrapolation for $\nu=2 / 5$, we can conclude that there is at most a small difference in values of $\Delta \epsilon^{\text {pol-sing }}$ for the both filling factors.

\section{APPENDIX B: EXCITATION MODES}

Here we present numerical data underlying sketches of Fig. 2 and briefly recall relevant data published before.

\section{Excitations from the polarized ground states}

In Fig. 6 (left panel) we put together the full spectra of several systems containing different number of electrons $N_{e}$ on a torus but having all the same filling factor $2 / 5$ and spin $S_{z}=N_{e} / 2$. The $\uparrow \uparrow \mathrm{GS}$ is taken as energy reference. We can distinguish a low-energy excitation mode with two distinct minima around 0.9 and 1.6 inverse magnetic length. Such a mode appears also in the spherical geometry, yet at higher energies (right panel). Recalling our experience with the $1 / N_{e} \rightarrow 0$ extrapolation, ${ }^{24}$ we believe that the torus data are closer to this limit and we take them as a base for the solid line of the upper panel of Fig. 2(a). The dispersion structure with two minima was found a long time ago (on a torus, ${ }^{26}$ sphere $^{38}$ and using projected ${ }^{27}$ or unprojected ${ }^{20}$ trial wave functions of CF magnetorotons) and the goal of Fig. 2 is to summarize all ED data accessible with present-day computers. Yet another approach would be to generalize results of Ref. 14 in the bosonization scheme of quasiparticle-pair excitations (see also remarks below).

The single-spin-flip excitations in Fig. 7(a) are the full spectra of the $S_{z}=N_{e} / 2-1$ subspace of $\nu=2 / 5$ again taken relative to the energy of the $\uparrow \uparrow \mathrm{GS}$. We find a well-separated low-lying excitation mode in agreement with CF magnetoroton trial wave functions. Although the difference between the torus and spherical geometry data is smaller than for the
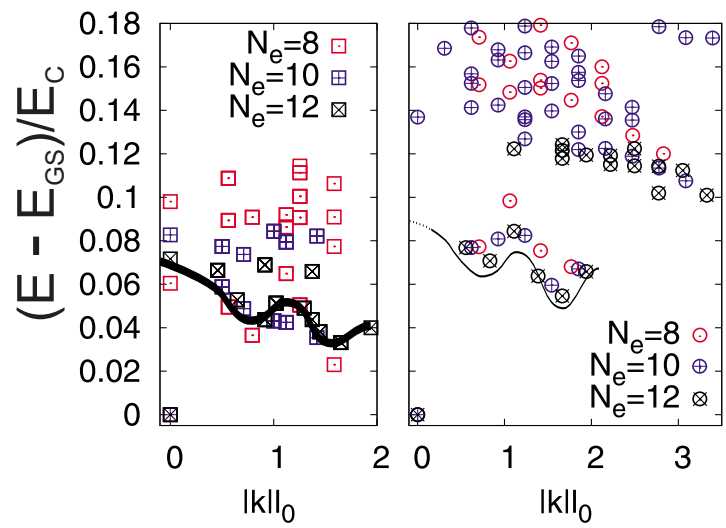

FIG. 6. (Color online) ED data for the $S_{z}=N_{e} / 2$ subspace of $\nu$ $=2 / 5$ with $N_{e}=8,10$, and 12 electrons on torus (left panel) and sphere [right panel; $2 Q$ used was 16,21 , and 26 , corresponding to the topological shift (Ref. 37) $\gamma=-4$ ]. The sketch of the no-spin-flip mode dispersion in Fig. 2(a) was drawn using the torus data (thick solid line). CF calculation in spherical geometry is shown for comparison on the right by a thin solid line (taken from Fig. 1 of Ref. 27). 

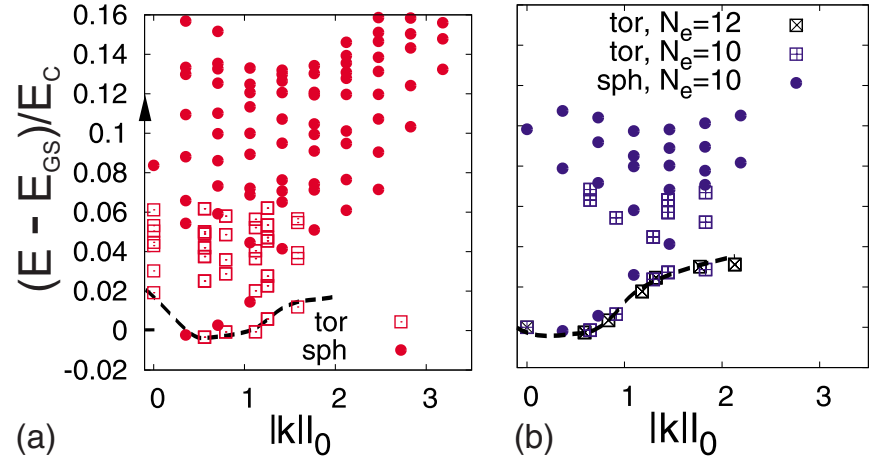

FIG. 7. (Color online) ED data for the $S_{z}=N_{e} / 2-1$ subspace of (a) $\nu=2 / 5$ with $N_{e}=8$ electrons and (b) $\nu=2 / 3$ with $N_{e}=10$ and 12 electrons. Differences between the torus and spherical geometry results are smaller than in Fig. 6, so that all data could be reasonably plotted in one viewgraph. The dashed lines were taken over to the sketches in Fig. 2(a). Values of $2 Q$ used were 16 (left) and 15 (right).

no-spin-flip excitations in Fig. 6, it also becomes sizable here, especially for larger values of $k \ell_{0}$. Because of sphere's curvature that will influence $\mathrm{QH} / \mathrm{QEr}$ pair energy when quasiparticle distance is larger, we again use the torus data for drawing the dashed line of the upper panel of Fig. 2(a).

Although in qualitative agreement, the spherical geometry ED energies are somehow larger relative to the CF trialwave-function result ${ }^{13}$ which, on the other hand, adheres more closely to the torus ED data in Fig. 7(a). In fact, the dashed guide-to-the-eye line in this graph lies close to the curve (a) of the lower panel of Fig. 1 in Ref. 13. It is not surprising because a square with periodic boundary conditions (torus) and a very large sphere (which can be used with trial wave functions) are both closer to the flat geometry than ED on a small sphere. Dispersion of this mode could also be calculated using the bosonized spin-wave operators ${ }^{14}$ within the Hamiltonian approach. ${ }^{39}$ Results of Doretto et al. ${ }^{14}$ would then have to be generalized to inter-CF LL excitations-at $\nu^{*}=2$, it applies to excitations both with and without spin flip as Fig. 1 suggests. This procedure lies beyond the scope of the present work but we nevertheless expect it to yield somehow larger energies than the ED does. Namely, the $\nu=1 / 3$ systems have effectively a factor of 3 smaller energies for the single-spin-flip mode (in zerothickness systems) (Ref. 24) than calculated in Ref. 14. According to experience with the Hamiltonian approach in general,${ }^{40}$ the discrepancy may tendentially stem from approximations underlying the bosonization procedure [Eq. (37) in Ref. 14].

The $\uparrow \uparrow$ GS of $\nu=2 / 3$ together with the full spectrum of the $S_{z}=N_{e} / 2$ subspace is particle-hole conjugate to and thus identical to $\nu=1 / 3$. The ED data for $\nu=1 / 3$ both for torus and for spherical geometry (see Fig. 5 of Ref. 24) can be condensed into the solid line on the lower panel of Fig. 2(a) which is the well-known magnetoroton mode. ${ }^{25}$ The singlespin-flip excitations at $\nu=2 / 3$ cannot be derived from this symmetry and the ED data of $S_{z}=N_{e} / 2-1$ are shown in Fig. 7(b). The dashed line in this viewgraph is replotted in the lower panel of Fig. 2(a). We are not aware of any published
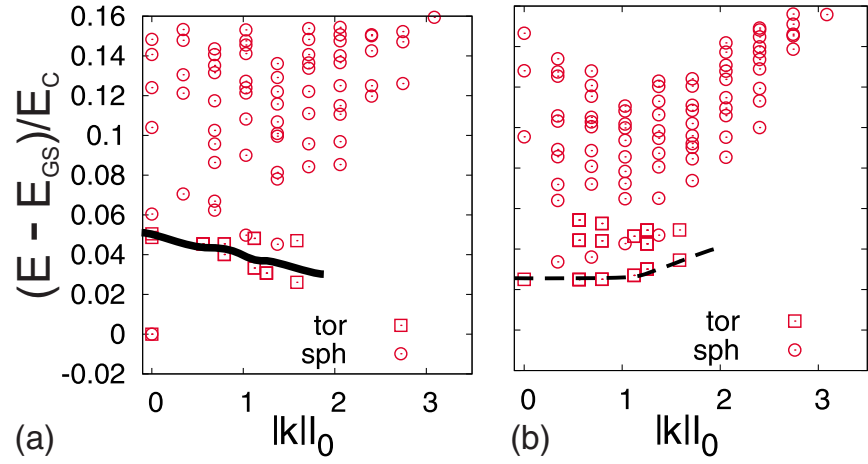

FIG. 8. (Color online) ED data for (a) the $S_{z}=0$ and (b) the $S_{z}$ $=1$ subspaces of $\nu=2 / 5$ with $N_{e}=8$ electrons. Torus and spherical geometry results are again plotted together in one viewgraph. The solid and dashed lines are replotted on the upper panel of Fig. 2(b). Values of $2 Q$ used were 17 for all spherical geometry calculations in this figure.

data from ED- or CF-based theories on this mode although it is of course not difficult to calculate. Neither spin-flip nor the magnetoroton excitations can be directly compared to results of Ref. 14 where only CF LL-index conserving excitations are considered.

\section{Excitations from the spin-inglet ground states}

For the no-spin-flip excitations we need to look into spectra of the $S_{z}=0$ subspace in Fig. 8(a) while the single-spinflip excitations corresponding to $S_{z}=1$ are shown in Fig. 8(b). Both spectra are plotted with the $\uparrow \downarrow$ GS of $\nu=2 / 5$ taken to have zero energy. These spectra suggest low-energy modes displayed by the solid (no spin flip) and dashed lines (single spin flip) which are redrawn in the upper panel of Fig. 2(b).

Finally, excitations from the $\nu=2 / 3$ spin-singlet GS are shown in the same manner in Fig. 9. The excitation modes indicated by the solid (dashed) lines lie somewhat higher than for $\nu=2 / 5$ and they are summarized in the lower panel of Fig. 2(b) as the no-spin-flip (single-spin-flip) mode. Both types of excitations have been somehow outside from the main focus of interest in the literature, probably because the spin-singlet GS are harder to come by experimentally, and
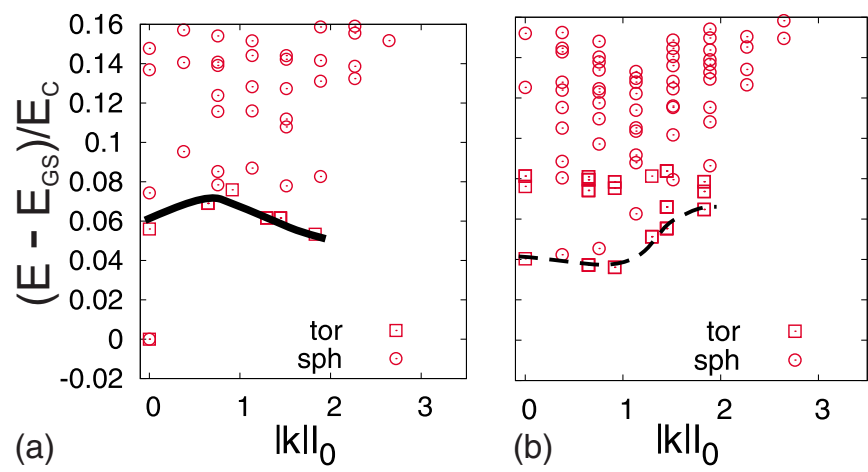

FIG. 9. (Color online) Same as Fig. 8 for $\nu=2 / 3$ and $N_{e}=10$ electrons (and $2 Q=14$ ). Solid and dashed lines are replotted on the lower panel of Fig. 2(b). 
(a)

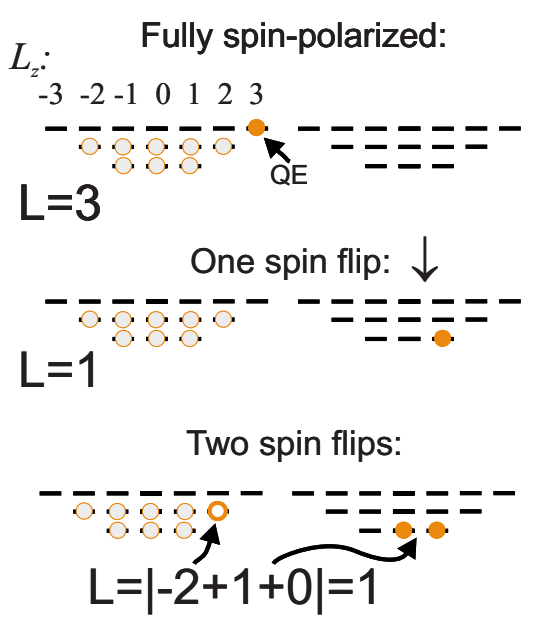

(b)
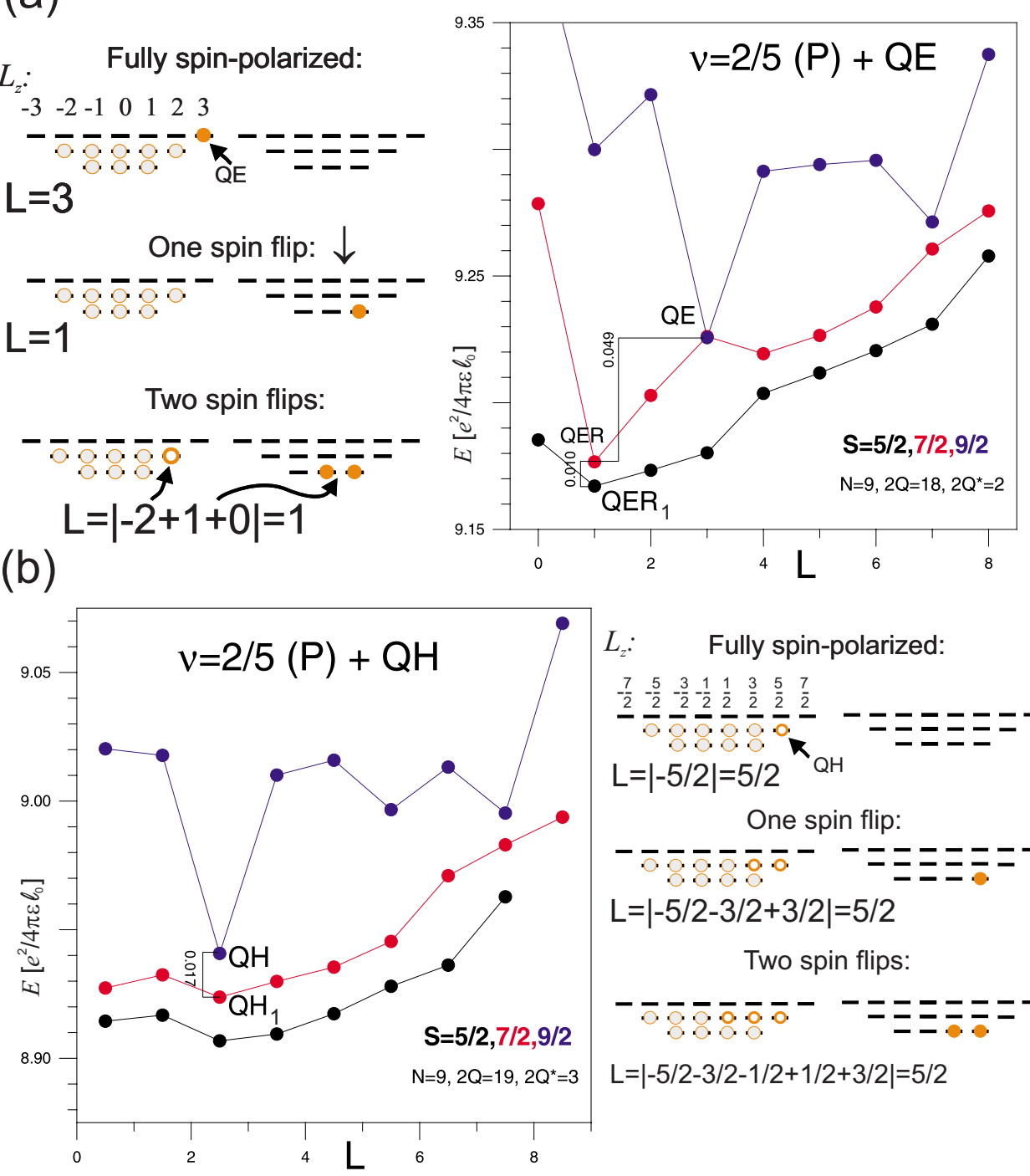

$L_{z}: \quad$ Fully spin-polarized:

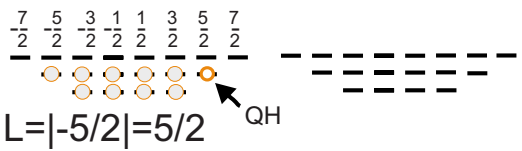

One spin flip:

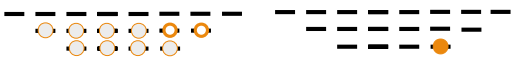

$\mathrm{L}=|-5 / 2-3 / 2+3 / 2|=5 / 2$

Two spin flips:

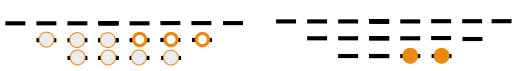

$L=|-5 / 2-3 / 2-1 / 2+1 / 2+3 / 2|=5 / 2$ we refer here the kind reader to the review of Chakraborty. ${ }^{35}$ Regarding the more recent work in this field, the Hamiltonian theory ${ }^{39}$ could be applied but again, first after the restriction to $\mathrm{CF}$ LL-index conserving excitations ${ }^{14}$ is relaxed.

\section{APPENDIX C: MULTIPLE-SPIN-FLIP STATES}

\section{Polarized ground states}

Figure 10 shows the spin-resolved spectra from the exact diagonalization of Eq. (2) for $N=9$ electrons on a sphere in a magnetic field corresponding to originally fully polarized $\nu$ $=2 / 5$ plus one QE or one QH. Symbolically, Fig. 10(a) shows 2/5(P)+QE while Fig. 10(b) shows 2/5(P)+QH. Blue (red, black) symbols show the lowest-energy state for each angular momentum $L$ and total spin $S=N / 2(N / 2$ $-1, N / 2-2)$, i.e., subspaces where we can look for quasiparticles with zero (one, two) spin flips on the background of the fully polarized state.

This magnetic field in terms of $2 Q$, number of magnetic monopole quanta placed in the center of the sphere, cannot be determined from the sole knowledge of $N$ and $\nu$ because $\nu=N /(2 Q+\gamma)$. The long-term experience with various FQHE systems is that the shift $\gamma$, which is a topological quantum number, ${ }^{37}$ can be often ${ }^{11}$ (though not always) correctly predicted using the NICF as follows. The degeneracy of the lowest (first, second) LL for CFs on a sphere is $\mid 2 Q^{*}$ $+1 \mid\left(\left|2 Q^{*}+3\right|,\left|2 Q^{*}+5\right|\right)$ if the effective magnetic field felt by the CFs corresponds to $2 Q^{*}$. So as to fill two lowest LLs completely and have one CF left for the third LL, as it corresponds to electronic filling factor of spin polarized $\nu$ $=2 / 5$ plus one $\mathrm{QE}$, we have to choose $2 Q^{*}=2$, see the top sketch on the left of Fig. 10(a). In order to pass over from the $\mathrm{CF}$ to electronic picture, we need to detach the two flux quanta per electron and get $2 Q=2 Q^{*}+2(N-1)$, i.e., $2 Q=18$ for $\nu=2 / 5(\mathrm{P})+\mathrm{QE}$.

Analogous chain of thoughts implies that for nine electrons, we must take $2 Q=19$ so as to get $\nu=2 / 5(\mathrm{P})+\mathrm{QH}$, as shown in Fig. 10(b). We can now read off the quasiparticle energies for Table II from Figs. 10 and 11 and moreover we present some basic interpretation of the states with more spin flips, which although not as deep as the skyrmion picture, ${ }^{9}$ suffices to establish that we use the correct values of $N$ and 
(a)

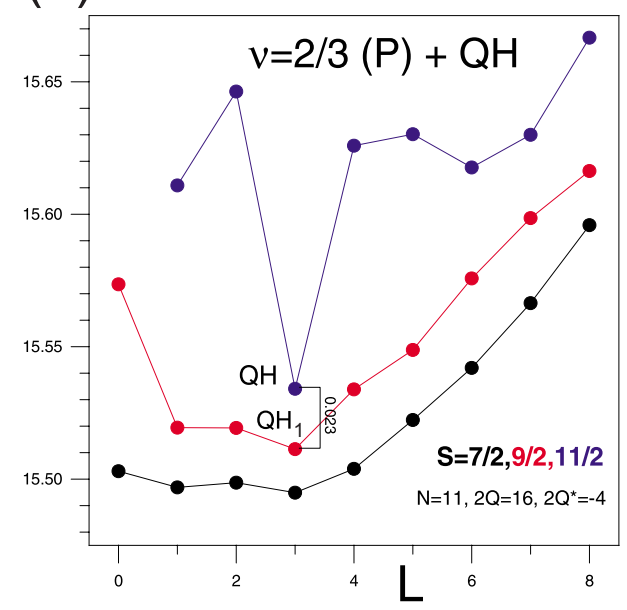

(b)

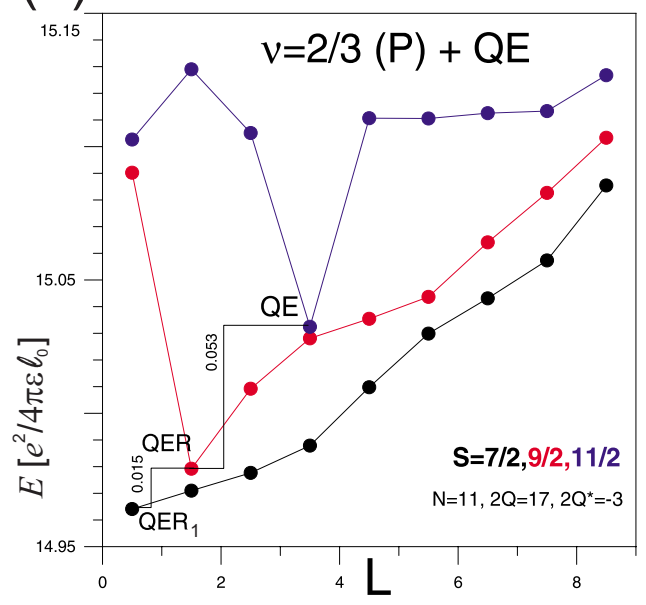

FIG. 11. (Color online) ED data for the $\nu=2 / 3$ polarized ground state plus one extra QE and $\mathrm{QH}$. (a)

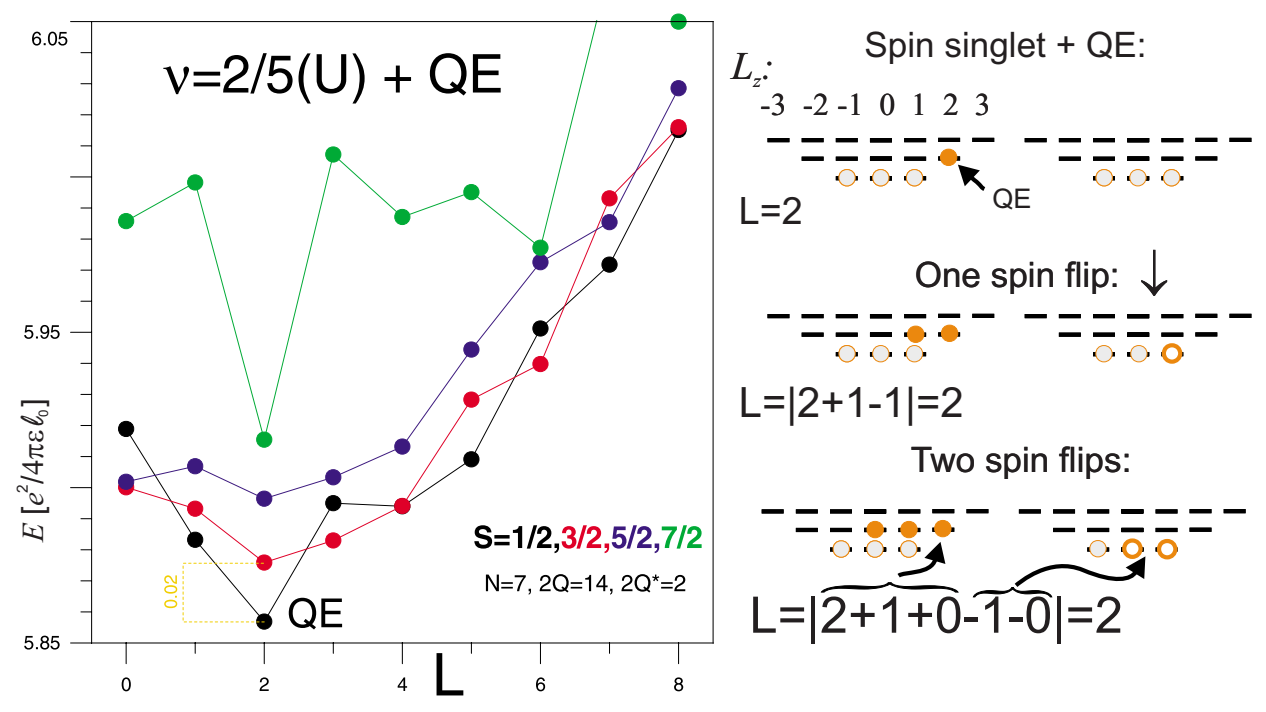

(b)

$L_{z}$ : $\quad$ Spin singlet $+\mathrm{QH}$ :

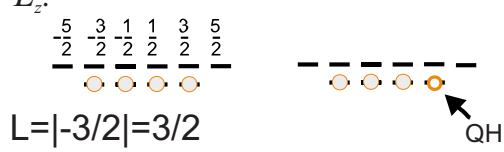

One spin flip:

- $\overline{\mathrm{O}} \overline{\mathrm{O}} \cdot \mathrm{O} \quad-\overline{\mathrm{O}} \overline{\mathrm{O}} \overline{\mathrm{O}}$

$L=|5 / 2-1 / 2-3 / 2|=1 / 2$

Two spin flips:

- - - : : $:-\bar{a}: \overline{0}$

$L=|5 / 2+3 / 2-3 / 2-1 / 2-(-1 / 2)|=5 / 2$

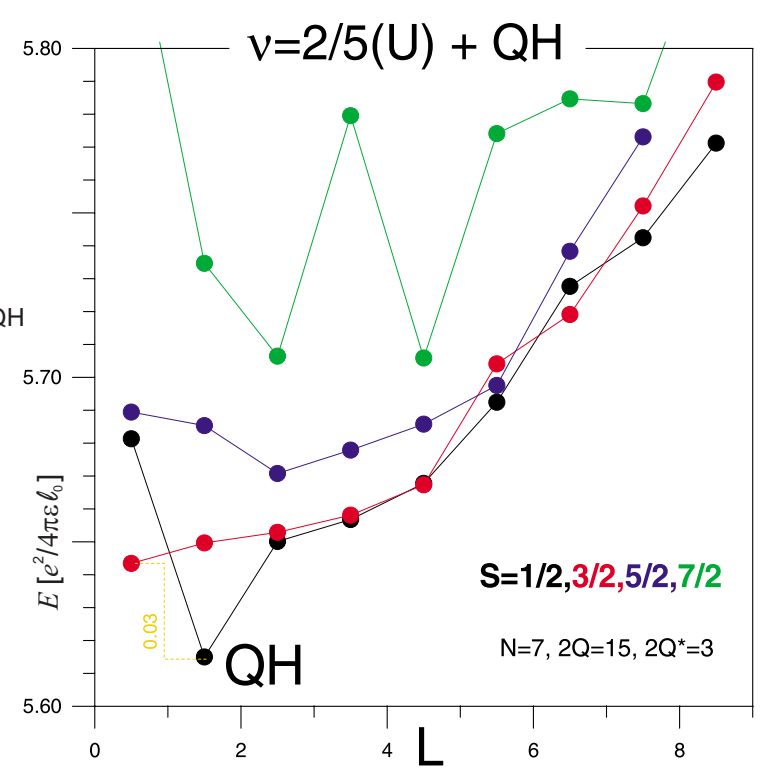

045407-11
FIG. 12. (Color online) The same as Fig. 10 for the $\nu=2 / 5$ spin-singlet ground state. 
(a)

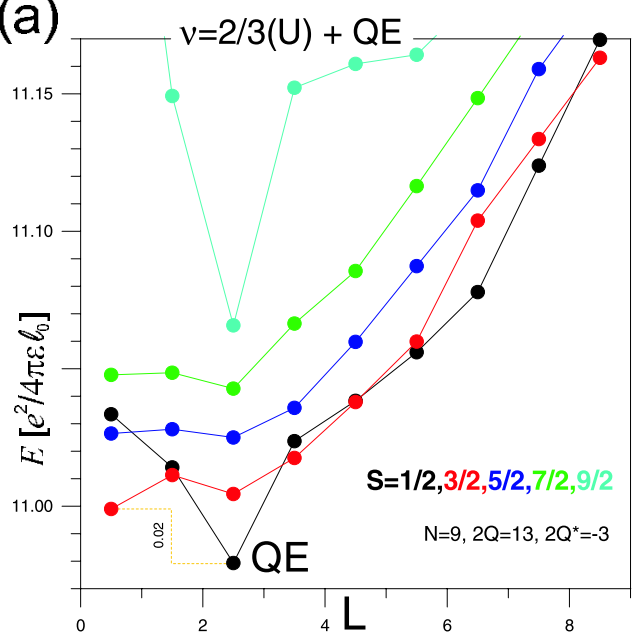

(b)

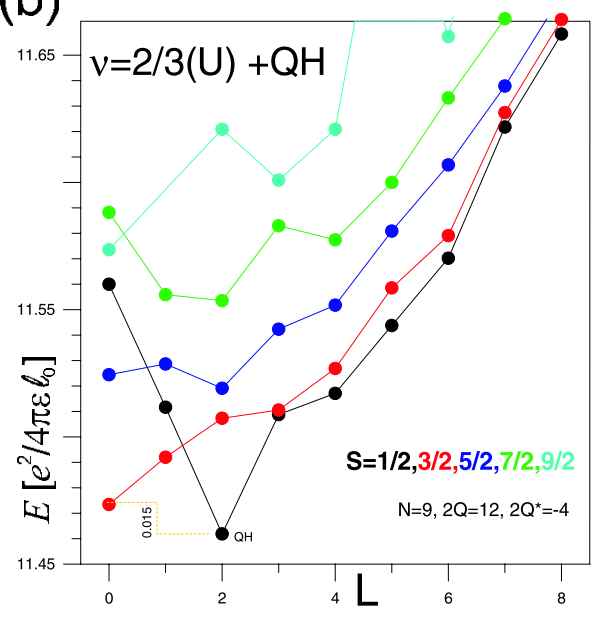

FIG. 13. (Color online) The same as Fig. 11 for the $\nu=2 / 3$ spin-singlet ground state.
$2 Q$ (i.e., choose the shift $\gamma$ correctly) to look for the states appropriate to $\nu=2 / 5$ and $2 / 3$.

Under the assumption that the uppermost sketch of Fig. 10 (a) correctly captures the structure of the energetically lowest FQH state of $2 / 5(\mathrm{P})+\mathrm{QE}$ and highest possible spin $S=N / 2$, the angular momentum of this state should be $L=3$ ( since completely filled shells have zero $L$ ) as observed in the numerical data. Similarly, the lowest $S=N / 2-1$ state corresponding to single spin flip from $2 / 5(\mathrm{P})+\mathrm{QE}$, shown in the middle sketch on the left of Fig. 10(a), should have $L=1$ as it indeed has.

Construction of the two-spin-flip state from $2 / 5(\mathrm{P})+\mathrm{QE}$ requires some additional information regarding the interaction between quasiparticles. Using the analogy of trions, we surmise that the quasiparticles, two quasielectrons in the $(0, \downarrow)$ CF LL, and one quasihole in the $(1, \uparrow)$ CF LL try to be spatially localized close to each other or assume in other words the states with highest $L_{z}$ ( $z$ component of $L$ ) possible within their LL as shown in the lowest sketch of Fig. 10(a). In this way, the total angular momentum of the three quasiparticles will be simply the sum of the individual angular quasiparticle angular momenta. This interpretation is supported by the lowest-energy state with two spin flips appearing at $L=|-2+1+0|=1$, see the ED data in Fig. 10(a). Note that quasihole angular momentum must be taken with opposite sign and the absolute value of the result should be taken.

Analogous arguments can again be implemented to calculate the expected $L$ of the lowest-energy state for $2 / 5(\mathrm{P})$ $+\mathrm{QH}$ and one or two spin flips. For all three cases, $S=N / 2$, $N / 2-1$, and $N / 2-2$, the sketches on the right of Fig. 10(b) turn out to predict the same value of $L$ as seen in the ED data on the left.

Argumentation with spin flips around $\nu=2 / 3(\mathrm{P})+\mathrm{QE}$ and $2 / 3(\mathrm{P})+\mathrm{QH}$ is largely identical and we only present the ED data in Figs. 11(a) and 11(b), respectively. The main difference to $\nu=2 / 5$ is that the effective field felt by the $\mathrm{CF}$ points in opposite direction to the real magnetic field, i.e., $2 Q^{*}$ is negative. In particular, $2 Q^{*}=-3$ for $2 / 3(\mathrm{P})+\mathrm{QE}$ and $2 Q^{*}=-4$ for $2 / 5(\mathrm{P})+\mathrm{QH}$.

\section{Singlet ground states}

Spin-singlet ground state at $\nu=2 / 5$ plus one $\mathrm{QH}$ or one QE will always have total spin of $S=1 / 2$. Quasiparticles with one (two) spin flips should therefore be located in the $S=3 / 2(S=5 / 2)$ subspace. ED data for these $2 / 5(\mathrm{U})+\mathrm{QE}$ and $2 / 5(\mathrm{U})+\mathrm{QH}$ systems are shown in Figs. 12(a) and 12(b) together with corresponding CF LL sketches.

Thus derived values of $L$ for $2 / 5(\mathrm{U})+\mathrm{QE}$ are in agreement with the ED results, $L=2,2$, and 2 for zero, one, and two spin flips, respectively. For two spin flips, however, the $L=2$ state energy already approaches that of the $L=0$ state. It is not surprising given that we study very small systems where two spin flips from $2 / 5(\mathrm{U})+\mathrm{QE}$ mean almost complete polarization of the system and thus a radical departure from the original incompressible singlet state being a background to a localized quasiparticle. The three-spin-flip system, $S=7 / 2$, in Fig. 12 is in fact already $2 / 5(\mathrm{P})+\mathrm{QH}$ for $N=7$ electrons.

The presented two-spin-flip data both for $2 / 5(\mathrm{U})+\mathrm{QE}$ and $2 / 5(\mathrm{U})+\mathrm{QH}$ should therefore be taken with caution. However, already the single-spin-flip data clearly demonstrate that, within the interpretation of the middle sketch on the right of Fig. 12(a), the interaction energy of the trionic complex cannot overcome the energy cost for promoting of a $\mathrm{CF}$ from the $(0, \downarrow)$ to the $(1, \uparrow)$ level.

The ED data for 2/3(U) $+\mathrm{QE}$ and $2 / 3(\mathrm{U})+\mathrm{QH}$ are shown in Figs. 13(a) and 13(b). All together we can state that the

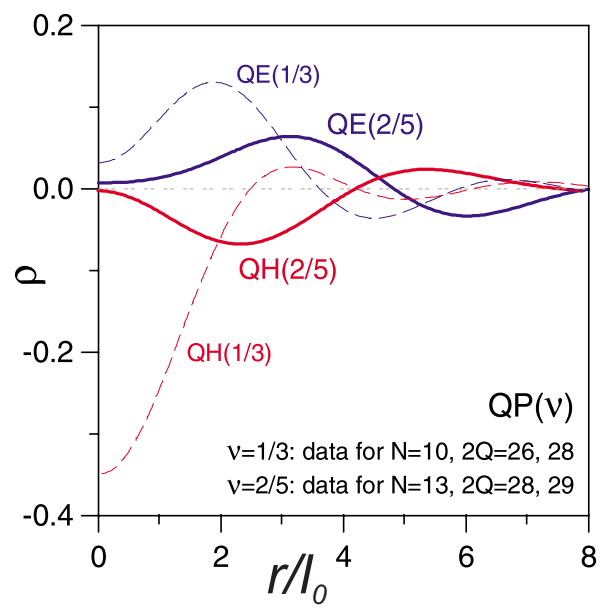

FIG. 14. (Color online) Profiles of charge densities of different quasiparticles at $\nu=1 / 3$ and $2 / 5$. 
angular momenta of the lowest-lying states with zero, one, and two spin flips are always those expected based on the sketches in Fig. 12 for $\nu=2 / 5$ and their analogous counterparts for $\nu=2 / 3$.

\section{APPENDIX D: QUASIPARTICLE CHARGE DENSITIES}

Although known from previous works, ${ }^{38,41}$ we show here the charge densities $\rho(r)$ for $\mathrm{QE}$ and $\mathrm{QH}$ at $\nu=1 / 3$ and $\nu$ $=2 / 5$. Eigenstates with one quasiparticle (quasihole) in the polarized GS on a sphere have nonzero $L$ and among them we always chose the one with $L_{z}=L\left(L_{z}=-L\right)$, i.e., the quasiparticle (quasihole) orbiting as close to the north pole as possible. The distances are measured along the big circle and the density is defined using the position operator of the $i$ th electron as the expectation value of $\rho(r)=\rho(|\vec{r}|)=-N_{e} \nu$ $+2 \pi \ell_{0}^{2} \nu \Sigma_{i} \delta\left(\vec{r}-\hat{r}_{i}\right), r=|\vec{r}|$ rather than as $2 \pi r \rho(r)$.

The charge densities in Fig. 14 can be used to interpret the no-spin-flip dispersion branches in Fig. 2(a). Note that QE $(\mathrm{QH})$ in the $\nu=1 / 3$ state is the same as $\mathrm{QH}(\mathrm{QE})$ of $\nu=2 / 3$ by virtue of the particle-hole symmetry.
${ }^{1}$ J. K. Jain, Phys. Rev. Lett. 63, 199 (1989).

${ }^{2}$ R. B. Laughlin, Phys. Rev. Lett. 50, 1395 (1983).

${ }^{3}$ D. C. Tsui, H. L. Stormer, and A. C. Gossard, Phys. Rev. Lett. 48, 1559 (1982).

${ }^{4}$ J. P. Eisenstein, H. L. Stormer, L. Pfeiffer, and K. W. West, Phys. Rev. Lett. 62, 1540 (1989).

${ }^{5}$ K. Park and J. K. Jain, Phys. Rev. Lett. 80, 4237 (1998).

${ }^{6}$ I. V. Kukushkin, K. v. Klitzing, and K. Eberl, Phys. Rev. Lett. 82, 3665 (1999).

${ }^{7}$ N. Shibata, Prog. Theor. Phys. Supp., 176, 182 (2008); N. Shibata and K. Nomura, J. Phys. Soc. Jpn. 76, 103711 (2007), and references therein.

${ }^{8}$ F. Schulze-Wischeler, E. Mariani, F. Hohls, and R. J. Haug, Phys. Rev. Lett. 92, 156401 (2004).

${ }^{9}$ S. L. Sondhi, A. Karlhede, S. A. Kivelson, and E. H. Rezayi, Phys. Rev. B 47, 16419 (1993).

${ }^{10}$ T. Jungwirth and A. H. MacDonald, Phys. Rev. B 63, 035305 (2000).

${ }^{11}$ K. Výborný, O. Certik, D. Pfannkuche, D. Wodzinski, A. Wojs, and J. J. Quinn, Phys. Rev. B 75, 045434 (2007), and references therein.

${ }^{12}$ T. Chakraborty, P. Pietiläinen, and F. C. Zhang, Phys. Rev. Lett. 57, 130 (1986); E. H. Rezayi, Phys. Rev. B 36, 5454 (1987); 43, 5944 (1991).

${ }^{13}$ S. S. Mandal and J. K. Jain, Phys. Rev. B 63, 201310(R) (2001).

${ }^{14}$ R. L. Doretto, M. O. Goerbig, P. Lederer, A. O. Caldeira, and C. Morais Smith, Phys. Rev. B 72, 035341 (2005).

${ }^{15}$ G. F. Giuliani and J. J. Quinn, Phys. Rev. B 31, 6228 (1985); Solid State Comm. 54, 1013 (1985).

${ }^{16}$ D. Lilliehöök, Phys. Rev. B 62, 7303 (2000).

${ }^{17}$ C. Kallin and B. I. Halperin, Phys. Rev. B 30, 5655 (1984).

${ }^{18}$ X. G. Wu, G. Dev, and J. K. Jain, Phys. Rev. Lett. 71, 153 (1993).

${ }^{19}$ F. D. M. Haldane and E. H. Rezayi, Phys. Rev. Lett. 54, 237 (1985).

${ }^{20}$ R. K. Kamilla, X. G. Wu, and J. K. Jain, Phys. Rev. Lett. 76, 1332 (1996).

${ }^{21}$ The debate about the form of CF interactions is not concluded at present. They were studied, for example, using ED [A. Wojs and J. J. Quinn, Phys. Rev. B 61, 2846 (2000); or A. Wojs, ibid. 79, 041304 (2009), and references therein] and Hamiltonian theories (Ref. 39); [M. O. Goerbig, P. Lederer, and C. Morais Smith, ibid. 69, 155324 (2004); or Phys. Rev. Lett. 93, 216802 (2004)] and discrepancies between the two approaches were found. While the ED should be here, in principle, the less approximative approach, it always suffers from the finite size of systems accessible to calculations; Other approaches are also conceivable [e.g., S. S. Mandal and J. K. Jain, Phys. Rev. B 66, 155302 (2002)] and some of them may, in principle, require to abandon the concept of two-body interaction between CFs.

${ }^{22}$ K. Výborný, Annalen der Physik, 16, 87 (2007), and references therein.

${ }^{23}$ F. D. M. Haldane, Phys. Rev. Lett. 55, 2095 (1985).

${ }^{24}$ A. F. Dethlefsen, R. J. Haug, K. Výborný, O. Čertík, and A. Wójs, Phys. Rev. B 74, 195324 (2006).

${ }^{25}$ S. M. Girvin, A. H. MacDonald, and P. M. Platzman, Phys. Rev. Lett. 54, 581 (1985).

${ }^{26}$ W. P. Su and Y. K. Wu, Phys. Rev. B 36, 7565 (1987).

${ }^{27}$ V. W. Scarola, Kwon Park, and J. K. Jain, Phys. Rev. B 61, 13064 (2000).

${ }^{28}$ S. S. Mandal and J. K. Jain, Phys. Rev. B 64, 125310 (2001).

${ }^{29}$ Mark Rasolt and A. H. MacDonald, Phys. Rev. B 34, 5530 (1986).

${ }^{30}$ S. E. Barrett, G. Dabbagh, L. N. Pfeiffer, K. W. West, and R. Tycko, Phys. Rev. Lett. 74, 5112 (1995); A. Schmeller, J. P. Eisenstein, L. N. Pfeiffer, and K. W. West, ibid. 75, 4290 (1995); E. H. Aifer, B. B. Goldberg, and D. A. Broido, ibid. 76, 680 (1996); D. K. Maude, M. Potemski, J. C. Portal, M. Henini, L. Eaves, G. Hill, and M. A. Pate, ibid. 77, 4604 (1996).

${ }^{31}$ T. Chakraborty, P. Pietiläinen, and R. Shankar, Europhys. Lett. 38, 141 (1997).

${ }^{32}$ A. Wójs and J. J. Quinn, Phys. Rev. B 66, 045323 (2002).

${ }^{33}$ F. Schulze-Wischeler, A. D. Dethlefsen, F. Hohls, U. Zeitler, and R. J. Haug, Phys. Status Solidi B 245, 409 (2008).

${ }^{34}$ A. Mielke, J. Phys.: Condens. Matter 2, 9567 (1990).

${ }^{35}$ T. Chakraborty, Adv. Phys. 49, 959 (2000).

${ }^{36}$ T. Chakraborty and F. C. Zhang, Phys. Rev. B 29, 7032 (1984); Very recently, the microscopic origin of the two ground states has become a topic of renewed debate [Maissam Barkeshli and Xiao-Gang Wen (unpublished)].

${ }^{37}$ Ch. Nayak and F. Wilczek, Nucl. Phys. B 455, 493 (1995).

${ }^{38}$ N. d'Ambrumenil and R. Morf, Phys. Rev. B 40, 6108 (1989).

${ }^{39}$ G. Murthy and R. Shankar, Rev. Mod. Phys. 75, 1101 (2003).

${ }^{40}$ R. Shankar, Phys. Rev. B 63, 085322 (2001).

${ }^{41}$ A. Wójs, D. Wodziński, and J. J. Quinn, Phys. Rev. B 74, 035315 (2006). 